\title{
PRP or not PRP? That is the question
}

\author{
Elizaveta Kon • Giuseppe Filardo
}

Published online: 16 April 2011

(C) Springer-Verlag 2011

Since the introduction of platelet concentrates as a topical adjuvant therapy to treat chronic leg ulcers in the late 1980, the use of platelet products has been extended to many fields of medicine, with the aim of promoting the healing process of various pathologies in numerous kinds of tissues [5].

Platelet-rich plasma (PRP) and other blood derivates, used alone or in combination with biomaterials, are under investigation in innumerable preclinical studies or even already used in clinical practice. Fields of application include dermatology, ophthalmology, dentistry, cosmetic, plastic and maxillofacial surgery, neurosurgery, urology, cardiothoracic surgery, as well as orthopedics, sport medicine, and recently, breakthroughs have been claimed even for the treatment of baldness. Looking more specifically into the orthopedic field, applications of PRP are numerous and heterogeneous: it has been used as adjuvant to promote the healing of bone defects and nonunions, bone fractures, laminectomy, spinal fusion, joint arthroplasty, bone implant osseointegration, and the treatment of various traumatic or degenerative pathologies of tendons, ligaments, muscle, and cartilage [2].

The rationale for using platelets in so many fields for the treatment of different tissues is that platelets constitute a reservoir of critical growth factors (GFs) and bioactive molecules that govern and regulate the healing process, which is quite similar in most tissues. Moreover, the attractive possibility of using patients' own growth factors, concentrated and in physiologic proportions, to enhance reparative processes in tissues with low healing potential,

E. Kon $(\bowtie) \cdot$ G. Filardo

Biomechanics Laboratory-III Clinic,

Rizzoli Orthopaedic Institute, Bologna, Italy

e-mail: e.kon@biomec.ior.it promising preliminary clinical findings, and their safety might explain the wide use of this biological approach [7].

However, although the popularity of PRP has reached a peak and there is lot of enthusiasm, talking, and advertising, it is realistic to say that nothing works for everything.

It is just not reasonable to believe that PRP is the solution for every medical problem, and its countless applications just show that often it is used indiscriminately, relying on the lack of side effects and on the fact that platelets take part in the physiologic healing process of the damaged tissue, thus assuming that if a few are good, then a lot may be better.

Deleterious effects have not been reported in clinical practice, yet, but GFs are potent molecules, and small variations in their concentrations can produce very different effects [8]. A bidirectional pattern has been observed for many molecules: the same GFs may present sub-optimal effects at low concentrations but paradoxically inhibitory or cytotoxic effects at high concentrations and potentially negative effects of PRP have also been suggested by recent preclinical observations [4]. Knowledge of the level of the applied platelet derived GFs is necessary to ensure a reliable and reproducible use of PRP, but only a few studies describe this aspect, and research into its proper dosage is complicated by the marked variations in GF concentrations even among individuals with similar platelet baseline counts.

Besides the appropriate dose, timing, number, and length of applications are other important factors that may influence the final outcome. Many other aspects may play a key role in the action mechanism of PRP and require further investigation [3]. Among these, cellularity is one of the most debated. In fact, not only platelets but also leukocytes, monocytes, macrophages, and mast cells are contained in many platelet concentrates. Some authors attribute better 
results to leucocyte depletion, because of the deleterious effects of proteases and reactive oxygen released from white cells; others consider them as a source of cytokines and enzymes, which may be important also for the prevention of infections. Their activation method also differs among different procedures. Thrombin and calcium have historically been used to activate platelets, but other methods have recently been chosen by other authors, using different factors or calcium chloride alone as a clot activator, or relying on the "in situ" collagen-mediated activation. The amount and speed of GF release may differ, and the molecules used may also influence the tissue with other mechanisms, thus leading to different results. Finally, factors specifically related to the microenvironment of the tissue involved and the type and phase of the pathology may also influence the results [5]. Another factor specific to the orthopedic field has to be considered; in fact, mechanical and biological stimuli may act in synergy, thus favoring the healing of musculoskeletal tissues.

Despite the promising preclinical findings and the huge interest in its clinical application, most of the questions on PRP remain unanswered. Indeed the literature contains more reviews than clinical studies, which consist mostly of a limited number of anecdotal reports or case series. More recently, some randomized controlled trials have being published, but results are still controversial $[1,6]$.

In this landscape, the analysis of the action mechanism and efficacy of PRP is complicated by the lack of standardization and the numerous factors that differ among the various procedures and may play an important role in biasing the results obtained.

PRP is an innovative promising approach, but biological studies to determine the action mechanisms of GFs administered with platelets and wide randomized well- designed studies are still needed to improve preparation methods and treatment modalities and clarify its real potential. While waiting for more robust evidence to support its use, the clinical applications of PRP and blood derivatives should be limited to well-controlled trials.

\section{References}

1. De Vos RJ, Weir A, van Schie HT, Bierma-Zeinstra SM, Verhaar JA, Weinans H, Tol JL (2010) Platelet-rich plasma injection for chronic Achilles tendinopathy: a randomized controlled trial. JAMA 303(2):144-149

2. Engebretsen L, Steffen K, Alsousou J et al (2010) IOC consensus paper on the use of platelet-rich plasma in sports medicine. $\mathrm{Br} \mathrm{J}$ Sports Med 44(15):1072-1081

3. Filardo G, Kon E, Marcacci M (2011) Reply to the letter by Dhillon and colleagues. Knee Surg Sports Traumatol Arthrosc. doi:10.1007/s00167-011-1413-1414

4. Kon E, Filardo G, Delcogliano M, Fini M, Salamanna F, Giavaresi G, Martin I, Marcacci M (2010) Platelet autologous growth factors decrease the osteochondral regeneration capability of a collagenhydroxyapatite scaffold in a sheep model. BMC Musculoskelet Disord 11:220

5. Kon E, Filardo G, Di Martino A, Marcacci M (2011) Platelet-rich plasma (PRP) to treat sports injuries: evidence to support its use. Knee Surg Sports Traumatol Arthrosc 19(4):516-527

6. Peerbooms JC, Sluimer J, Bruijn DJ, Gosens T (2010) Positive effect of an autologous platelet concentrate in lateral epicondylitis in a double-blind randomized controlled trial: platelet-rich plasma versus corticosteroid injection with a 1-year follow-up. Am J Sports Med 38(2):255-262

7. Tschon M, Fini M, Giardino R et al (2011) Lights and shadows concerning platelet products for musculoskeletal regeneration. Front Biosci (Elite Ed) 3:96-107

8. Weibrich G, Hansen T, Kleis W, Buch R, Hitzler WE (2004) Effect of platelet concentration in platelet-rich plasma on periimplant bone regeneration. Bone 34(4):665-671 